\title{
Progression: von „Low Load" zu „High Load”
}

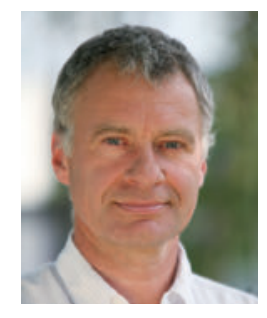

Hans-Josef Haas

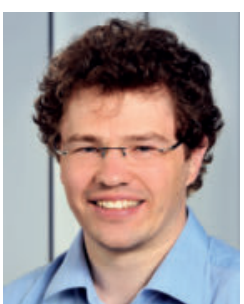

Eduard Kurz
Korrespondenzadresse

Hans-Josef Haas

spt-education

Andreas-Schlüter-Str. 15

53639 Königswinter

hjhaas@spt-education.de

www.spt-education.de

Bibliografie

DOI https://doi.org/10.1055/a-0965-9141

Sportphysio 2020; 8: 1

(c) Georg Thieme Verlag KG Stuttgart · New York

ISSN 2196-5951
„Train hard but smart” lautete der Titel eines spannenden Artikels von Tim Gabbett im BJSM [1]. Unter anderem dieser Beitrag war Anlass für dieses Schwerpunktheft „Progression“. Dabei weist der Slogan auf eine altbekannte Weisheit hin: Wollen wir Strukturen stärken, muss das Training klug und intensiv gestaltet sein. Zu hartes Training mit zu kurzen Pausen führt zum Abbau der Strukturen, weil die Zeit für die Regeneration zu kurz ist. Sind die Trainingsreize zu schwach, sei es aufgrund von Krankheit, Inaktivität oder auch Unterdosierung, erfolgt ebenfalls eine Deadaptation im Sinne einer Reduzierung der Proteinmasse mit funktionellen Einbußen. Die Balance zwischen den Extremen zu viel oder zu wenig Training zu finden, bezeichnet Gabbett als „smart“.

In den zurückliegenden Jahren wurden in der Rehabilitation von orthopädisch-traumatologischen Verletzungen am Bewegungsapparat neben den „zeitbasierten“, also den am zeitlichen Verlauf der Wundheilungsphasen orientierten Vorgehensweisen verschiedene Konzepte der „kriterienbasierten“ Rehabilitation etabliert. Dabei verfolgen die letztgenannten die Idee, nach dem „Wenn-dannPrinzip“ zu verfahren: Wenn ein bestimmtes Kriterium im Rehabilitationsprozess erfüllt ist, dann kann der nächste Schritt erfolgen.

Eine Problematik dieser Konzepte ist, dass in der Rehabilitation von Sportverletzungen die relevanten Kriterien bisher nur für eine geringe Anzahl von Verletzungen evaluiert werden konnten. Dies macht ein einheitliches zeitliches und inhaltliches Vorgehen im Return-to-Competition-Prozess (RTC-Prozess) für unterschiedliche Bedingungen unrealistisch oder zumindest sehr schwierig. Immer wieder stellt sich die Frage, wann oder wie die nächste Progression im RTC-Prozess erfolgen soll.
Im vorliegenden Schwerpunktheft haben wir versucht, Möglichkeiten der Belastungsprogression von „Low Load“ zu „High Load“ aufzuzeigen. Frank Diemer trägt Hintergrundwissen, Definitionen und wichtige Ziele von Progressionen im RTC-Prozess zusammen und belebt seinen Beitrag mit Beispielen aus der Praxis. Christian Szepessy Steinmann und Hans-Josef Haas konzentrieren sich in ihrem Beitrag auf die Progressionsmöglichkeiten, die in einer systematischen Reihenfolge verschiedener Trainingsmethoden für Kraft, Schnelligkeit, Ausdauer und Koordination liegen. Ralf Roth schließlich verknüpft am Beispiel eines Stufenmodells im Aufbautraining nach einer Verletzung der unteren Extremität ein zeitbasiertes Stufenmodell und einen funktionsbasierten Algorithmus im RTC-Prozess, um mögliche Progressionen aufzuzeigen.

Wir wünschen allen Lesern im Namen unseres Herausgeberteams viel Spaß und Erkenntnisgewinne bei der Lektüre.

Hans-Josef Haas und Eduard Kurz

\section{Literatur}

[1] Gabbett T]. The training-injury prevention paradox: Should athletes be training smarter and harder? Br J Sports Med 2016; 50: 273-280 